\section{$\underset{\substack{\text { hommes } \\ \text { \& migrations }}}{ }$}

\section{Hommes \& migrations}

Revue française de référence sur les dynamiques

migratoires

$1301 \mid 2013$

Migrations et mondes ruraux

\title{
La MAPEmonde
}

L'accueil des personnes étrangères dans les Hautes-Alpes

\section{Karine Moreau}

\section{Q OpenEdition \\ 1 Journals}

\section{Édition électronique}

URL : http://journals.openedition.org/hommesmigrations/1947

DOI : 10.4000/hommesmigrations. 1947

ISSN : 2262-3353

\section{Éditeur}

Musée national de l'histoire de l'immigration

\section{Édition imprimée}

Date de publication : 1 janvier 2013

Pagination : 174-177

ISBN : 978-2-919040-21-6

ISSN : $1142-852 X$

\section{Référence électronique}

Karine Moreau, "La MAPEmonde », Hommes \& migrations [En ligne], 1301 | 2013, mis en ligne le 29 mai 2013, consulté le 22 septembre 2020. URL : http://journals.openedition.org/hommesmigrations/ 1947 ; DOI : https://doi.org/10.4000/hommesmigrations.1947

Ce document a été généré automatiquement le 22 septembre 2020.

Tous droits réservés 


\title{
La MAPEmonde
}

\author{
L'accueil des personnes étrangères dans les Hautes-Alpes
}

\author{
Karine Moreau
}

\section{NOTE DE L'ÉDITEUR}

La MAPEmonde (Mission d'accueil des personnes étrangères) est un service créé par la MJC-Centre social du Briançonnais, dirigée par Luc Marchello, pour répondre aux besoins des personnes étrangères. Bien que son siège soit à Briançon, ses deux conseillères juridiques et sociales sillonnent le département des Hautes-Alpes. Contact : MJC-Centre social du Briançonnais 35, rue Pasteur, 05100 Briançon

\section{Des immigrés dans les montagnes}

1 Le département des Hautes-Alpes, en région Paca, a une physionomie toute particulière : ce pays de montagnes (Briançon est considéré comme la ville la plus haute d'Europe), faiblement peuplé, s'est développé autour des sommets enneigés des stations de ski et du massif des Écrins. Si le col de Montgenèvre marquant la frontière officielle avec l'Italie est un lieu important de passage de migrants, l'arrivée de personnes étrangères allant s'installer dans nos montagnes a aujourd'hui changé le visage de la région.

2 Ici, en matière d'immigration, le hasard n'existe pas vraiment. Il faut avoir une bonne raison pour venir dans cette partie de France, ne pas craindre le froid ni l'altitude, et penser qu'on peut y construire un avenir. Au cours de ces dernières années, seules deux personnes nous ont offert la surprise de passer par chez nous sans y avoir été invitées : un Lituanien qui avait réussi à passer la frontière de Montgenèvre, et qui faisait halte pour se soigner les pieds après avoir parcouru plus de 2000 kilomètres pour rejoindre une grande ville française, et un Somalien mal orienté à la gare SNCF par un passeur. Pensant débarquer à Paris avec son petit bout de papier estampillé d'une adresse 
sacrée, il est arrivé en plein hiver à Briançon. Après une nuit en foyer d'urgence, il a repris le bon train vers Paris. Pour les autres, en majorité des Maghrébins, des Turcs et des Européens, Briançon et ses alentours représentent un espace familial, une opportunité de travail dans le bâtiment ou dans le tourisme, un lieu où l'on vient se soigner puis s'installer pour y bénéficier du bon air. Les arrivées sont donc prévues et un tant soit peu organisées (notion toute culturelle et personnelle) avec les contrats de main-d'œuvre étrangère de l'office français de l'immigration et de l'intégration (OFII), le regroupement familial, le mariage avec un Français... De plus, la majorité des personnes que nous accueillons viennent d'un milieu rural où le froid et la neige ne sont pas inconnus (Atlas marocain, mont Erciyes en Turquie, Patagonie...). Elles apprécient les endroits tranquilles où les relations avec les autochtones paraissent plus simples et plus chaleureuses.

3 L'immigration maghrébine, notamment dans le bassin de l'Argentière-la-Bessée, remonte principalement aux années 1960 avec le recrutement massif dans l'usine d'aluminium Pechiney. À sa fermeture en 1988, l'Association d'entraide pour le développement (AEDA) aida les personnes étrangères sur le plan professionnel et pour les divers aspects de la vie sociale et administrative. Dissoute au début des années 2000 pour raisons budgétaires, cette association a laissé un grand vide. Si le département n'est pas connu pour son taux d'immigration flamboyant, environ 3500 personnes étrangères n'en sont pas moins dans les Hautes-Alpes avec des problématiques complexes encadrées par une toile législative changeante. Aussi a-t-il été nécessaire de créer une mission d'accueil adaptée au territoire pour informer et accompagner les personnes étrangères dans l'accès au droit et impulser une dynamique d'accueil départementale.

4 La MJC-Centre social du Briançonnais s'est toujours impliquée dans une réflexion et dans des actions culturelles et sociales visant à dynamiser le territoire. Aussi s'est-elle logiquement emparée de la problématique de l'accueil et de l'accompagnement des personnes étrangères en lien avec les structures et les habitants du département. L'idée est donc de leur offrir un espace d'accueil répondant à leurs préoccupations spécifiques tout en ayant à l'esprit la notion d'intégration: comment le département peut-il se mobiliser pour accueillir activement des personnes venues d'horizons divers et les intégrer dans un projet commun, tout en respectant et valorisant leurs apports particuliers? Un grand projet, également partagé par les relais de services publics maillant le territoire, qui tente de combiner une idée large d'accès aux services et la proximité avec la population. La MAPEmonde revendique sa place d'épicerie-bar des papiers, loin des centres urbains. Il n'est pas toujours facile, avec cette configuration originale, de se faire reconnaître en ville, d'autant plus que les Gapençais doutent souvent qu'il y a le même nombre de kilomètres entre Briançon et Gap qu'entre Gap et Briançon!

\section{L'accueil, sac à dos}

5 Les professionnels des structures sociales et administratives des Hautes-Alpes, occasionnellement confrontés à l'accueil des personnes migrantes, ne sont pas particulièrement informés sur le droit des étrangers. Aussi nous sommes chargés de centraliser l'information en créant un centre de ressources concrétisé par un bureau, deux ordinateurs, quelques livres et deux conseillères juridiques et sociales. Celles-ci, 
branchées continuellement sur Internet, s'échappent régulièrement des montagnes pour se nourrir au chaudron de la ville et rapporter des nouvelles fraîches. Les sacs à dos remplis, nous retransmettons ensuite ces informations et en les adaptant à notre contexte, par des cycles de formation destinés aux acteurs professionnels, des réunions thématiques, des mailing list, des conférences ou divers événements grand public. Ainsi, les travailleurs sociaux n'hésitent pas à nous solliciter lorsqu'ils reçoivent des migrants pour trouver des informations et des conseils adaptés à leur demande.

Nous accueillons directement le public étranger au cours de permanences dans différentes communes et espaces d'accueil du département: Briançon (MJC-Centre social, Maison de la justice et du droit), L'Argentière-la-Bessée, Embrun, Laragne (Relais de services publics) et Gap (Point d'accès au droit). Ces permanences permettent à la fois d'être proche du public et de s'imprégner du contexte local et des ressources mobilisables afin d'offrir une réponse sur mesure. Dans cette optique, nous n'hésitons pas à accompagner les gens dans les structures concernées par leur demande. Nous apprécions aussi les invitations à domicile qui permettent de modifier les rôles déterminés par l'architecture du bureau. Ce sont nos lieux de formation favoris. Merci à ceux qui nous offrent le luxe de ces conditions de travail.

7 En quoi consiste la spécificité de notre travail en milieu rural ? La faible démographie n'est pas synonyme de similarité de problèmes. Au contraire, des situations fort diverses se présentent, auxquelles nous sommes rarement préparés. Cela offre l'avantage de limiter la routine, mais oblige également à un questionnement et une actualisation permanents de nos compétences. Le travail effectué pour répondre à un cas particulier n'est pas toujours réutilisable. La faible présence de structures spécialisées comme il en existe en ville (traducteurs, avocats spécialisés en droit des étrangers, Centre national d'information sur les droits des femmes et des familles, centre de médiation ethnoclinique, dispositifs scolaires d'accueil des enfants non francophones...) nous impose d'ouvrir largement la porte aux différentes problématiques, afin que les personnes puissent trouver un soutien nécessaire, laissant les frontières de nos actions relativement mouvantes pour nous-mêmes, nos partenaires sociaux et le public. Ainsi, la MAPEmonde accompagne les personnes étrangères (et quelques Français pour ne pas paraître discriminatoire!) pour un problème de titre de séjour, de difficultés scolaires, de vaccination d'enfants, de violences conjugales, de recherches de disparus pendant la guerre d'Algérie, de retraite de gens rentrés au pays, de besoin de lien social, de courrier à expliquer, de billets d'avion à réserver, de recours pour refus de visa... Nous avons reçu un vieux couple d'Algériens dont la mésentente spectaculaire empêchait toute démarche. Les rendezvous tournaient à la dispute et toute intervention s'est révélée vaine. C'est une Algérienne qui nous connaît bien et qui nous sollicite régulièrement qui leur a conseillé de venir nous voir, parce que nous travaillons à "aider les immigrés". Ainsi, les professions se mêlent, au risque d'un bricolage permanent.

\section{En quête de chaleur humaine}

8 Deux constats ont entraîné l'élargissement de nos actions. Tout d'abord, nous avons remarqué que certaines personnes prétextaient d'avoir des papiers à régler pour venir nous voir, laissant entrevoir une certaine solitude et un besoin de lien social. Ensuite, régulièrement, des gens sont étonnés de voir un service dédié aux personnes 
étrangères et ils veulent entrer en contact avec nous. Aussi avons-nous mis en place des actions ponctuelles avec un objectif double : susciter la rencontre avec les populations locales et rendre visible la présence des familles étrangères. Ces actions sont mises en place en fonction des opportunités et des besoins.

Le "café-papiers", s'il n'a pas encore trouvé sa forme aboutie et connaît de constantes transformations, est un espace convivial et ponctuel qui permet d'échanger collectivement sur un thème. Pensé prioritairement pour les personnes étrangères, il est ouvert à tous. L'idée est d'accueillir un intervenant et d'échanger avec lui autour d'un café, pour affiner nos connaissances sur la structure qu'il représente, désacraliser l'institution, permettre à cet intervenant de développer des liens avec les personnes étrangères. Ainsi avons-nous accueilli des intervenants sur la retraite, la nationalité française, la formation professionnelle, les discriminations, les organismes sociaux... Les retours des intervenants et des personnes étrangères nous encouragent à poursuivre ce travail qui permet aux immigrés de s'adresser avec plus de confiance aux institutions. Actuellement, les cafés-papiers sont intégrés dans les cours de "français langue d'intégration" organisés pour les primo-arrivants par le Groupement d'établissements publics d'enseignement (Greta).

À la MAPEmonde, qui fait partie de la MJC-Centre social du Briançonnais, nous incluons les personnes rencontrées dans les actions des différents services : sorties à ski avec le référent-famille - un groupe de femmes turques portant pantalons et lunettes de soleil pour la première fois et descendant des pistes sur les fesses ont provoqué des fous rires collectifs - , soirée ciné-débat-concert pour sensibiliser la population locale aux réalités vécues par les Roms, repas sortis du sac, soirées culturelles, orientation vers des activités culturelles ou sociales (danse, cours d'informatique, accompagnement scolaire, cours de français organisés par des bénévoles...), accompagnement d'un projet de mise en scène théâtrale mêlant immigrés et Français. L'important, même si cela reste discret, est la chaleur des liens qui se développent et qui font aborder l'immigration sous un angle affectif. L'objectif est considéré comme pleinement atteint lorsque des relations amicales se nouent.

11 Enfin, un autre axe est développé : la valorisation de l'apport de l'immigration dans le nord des Hautes-Alpes. Plusieurs documents ont été produits et diffusés, mettant en valeur la présence étrangère dans les Hautes-Alpes. Un livre sur l'implication des travailleurs étrangers dans le bâti briançonnais est en cours. L'objectif étant que les Briançonnais sentent la richesse des histoires vécues par ces ouvriers exilés derrière les pierres et le bitume.

\section{Avantages et inconvénients du travail en montagne}

12 Le travail auprès des personnes étrangères dans nos montagnes offre plusieurs avantages. En raison du faible nombre d'étrangers, le temps d'accueil n'est pas limité par la file d'attente (bien que cela puisse arriver!) et nous avons le sentiment de pouvoir nous adapter aux besoins de tous. Cette disponibilité dépasse le cadre du bureau car la petite taille des villes ou villages repousse les frontières de la vie privée. Ainsi, faire son marché ne se limite pas à faire approvisionner la famille, c'est également l'occasion de poursuivre les échanges "professionnels". Certes, cela donne une impression de travail continu, mais cela offre aussi l'opportunité de créer des liens 
différents. Et que c'est bon, quand le temps est glacial, de se voir invité à boire un café chaud par des chibanis et de passer un bon moment à rire de la vie !

13

Cependant, l'éloignement, l'isolement et le confinement apportent leur lot de soucis. Un sentiment de solitude peut se développer, notamment lorsque nous sommes informés de la richesse des réflexions offertes par les villes, en termes de formation, conférences, rencontres... et qu'il ne nous est pas possible de nous y rendre. Alors, nous tentons de faire venir des intervenants sur place, comme des militants associatifs ou des chercheurs spécialisés dans l'immigration, mais cela engendre un autre stress : il faut se brancher sur Météo France en continu et implorer les dieux de la neige de laisser notre intervenant venir jusqu'à nous sans encombre.

Enfin, notre travail en altitude donne un côté exotique à notre mission, il nous arrive parfois de nous demander si nous sommes pris au sérieux, d'autant que l'immigration n'est pas vécue ici comme un problème médiatisé. Et c'est justement ce que nous aimerions voir continuer.

\section{AUTEUR}

\section{KARINE MOREAU}

Conseillère juridique, et l'équipe de la MAPEmonde 